\title{
OPTIMAL FLOCK STRUCTURE OF PIG FARM PROVIDING MINIMUM COSTS
}

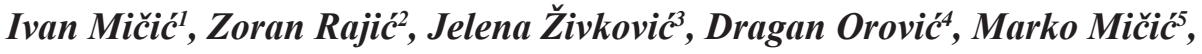 \\ Ivana Mičićc ${ }^{6}$ Marija Mičić ${ }^{7}$
}

\begin{abstract}
The study deals with the analysis of production costs, as well as the net area in the production of the herd of organizational structures at the pig farm. The question arises of the optimum production structure in the cooperative household, which is mainly oriented to the production of Pigmeat. In this paper, a concrete model of linear programming for the optimal organizational structure of the herd of pig farms of the net effective area was defined. The research covered the cooperative farm "1.Decembr" in Žitorađa in Toplički district. For 2013, the production technology of agricultural animals was followed, and economic results were analyzed. In the observed period of one year. It was found that there were 28,252 throats on the farm. Total agricultural growth in 2013 amounted to $664,920 \mathrm{~kg}$, in the amount of 781,569.7 €. Total death: piglets on wolves 6,315 throats, stuffed pigs 3,425 throats, $17,150 \mathrm{~kg}$, tooth 928 throats, 40,980 kg, pigs 67 throats 13,930 $\mathrm{kg}$, immature 3 throat $890 \mathrm{~kg}$
\end{abstract}

Key words: crop rotation, surface area by throat, increment, death, minimum costs

JEL: $Q 12, Q 13$

1 Ivan Mičić M.A., Ph.D. student, University in Belgrade, Faculty of Agriculture, Nemanjina street no. 6, 11080 Zemun, Serbia, Phone: +381 1126153 15, E-mail: divanlav@gmail.com

2 Zoran Rajić Ph.D., Full Professor, University in Belgrade, Faculty of Agriculture, Nemanjina street no. 6, 11080 Zemun, Serbia, Phone: +381 1126153 15, E-mail:zorajic@agrif.bg.ac.rs

3 Jelena Živković M.A., University in Belgrade, Faculty of Agriculture, Nemanjina street no. 6, 11080 Zemun, Serbia, Phone: +381 6387882 51, E-mail:zivkovic@jaxoo.com

4 Dragan Orović M.A., Ph.D. student, University in Belgrade, Faculty of Agriculture, Nemanjina street no. 6, 11080 Zemun, Serbia, Phone: +381 6987025 20, E-mail: draganorovic@gmail.com

5 Marko Mičić, graduate economist, University of Niš, Faculty of Economics, Trg Kralja Aleksandra Ujedinitelja no. 11, Niš, Serbia, Phone: +381 63680 040, E-mail: markomicic89@gmail.com

6 Ivana Mičić M.A, Ph.D. student, University of Niš, Faculty of Economics, Trg Kralja Aleksandra Ujedinitelja no. 11 Niš, Serbia, Phone: +381 63233 603, E-mail: ivancica@gmail.com

7 Marija Mičić M.A, Ph.D. student, University of Niš, Faculty of Technology in Leskovac, Bulevar Oslobođenja no. 124, Leskovac, Serbia, Phone: +381 6286745 98, E-mail: marija84micic@gmail.com

EP 2017 (64) 3 (1003-1018) 


\section{Introduction}

Regardless of the market-oriented pig production, it is very difficult to provide a detailed insight into the poultry farm's turnover in pig production, which is the basis of the research in the work and proving its net effective surface area of the main structure in the production process. (Andrić, 1998; Mičić et al., 2016a) find that with the increase in the number of deciduous piglets by sows from 10 to 20 heads of pigs, the price of piglet is reduced by $79.09 \%$, while the costs of sows increase by $11.67 \%$ per year. (Džinić et al., 2003; Petrović et al., 2010; Mičić, 2014) states that the improvement of the genetic basis of pigs is an indispensable prerequisite for achieving the highest intensity in this branch of livestock production.(Petrovićet al., 2010; Vidovic et al.,2012) state that the annual genetic progress for daily gain was $8-11 \mathrm{~g}$, for food conversion between 0.03 and $0.05 \mathrm{~kg}$, as well as $0.35-1.00 \%$ for meat content in Hemispheres. (Tomović et al., 2005; Mičić et al., 2016b) find that in the foreseeable future, on the basis of previous research and results in practice, there are opinions that the production of freight can be expected to have much better results as well: The framework organizational structure should move within the following limits : Piglets $20-25 \%$, breeding fowls $5-8 \%$, fattening pigs $70-80 \%$, sows $4-8 \%$ and non-fennel 0,3-5\%.(Radovic, 2002;Rajić, 2003; Mičić et al., 2016b) state that the next space is planned for an individual of certain categories:piglets on cheese $0,4 \mathrm{~m}^{2}$, breeding pig $0,7 \mathrm{~m}^{2}$, pig fodder $1,2 \mathrm{~m}^{2}$, sowers 1,5 $\mathrm{m}^{2}$ and nests $1,8 \mathrm{~m}^{2}$. The costs of certain categories of holding are as follows: for a pig 700 dinars, piglets for breeding (breeding subspecies) 1,800 dinars, pig meat 3,500 dinars, sowers 5,000 dinars and non-salty 4,000 dinars. The same author states that "The specificity of livestock production is conditioned by the fact that in addition to the final line of livestock production ("final categories "), there are several other categories. There are two possible variants in defining livestock variables. The first implies that each category of livestock represents a separate activity and is expressed by a physical or conditional number of throats, While the second variant predicts that in the model only the final category of a particular livestock is figured, and that the necessary inputs and predicted outputs of the accompanying categories cumulatively point out through the final category. (Živković, Perunović, 2012; Petrović et al., 2013; Zekić et al., 2013; Mičić et al., 2016a) They conclude that studies related to the economic parameters of pig production are related to costs in the first stage of the production process and the determination of the total costs of individual categories of pigs they hold using the optimal organizational structure of rotation of crops on the farm, which provides minimal costs.

\section{The aim of the research}

In proofing and research, a scientific method was first used, whose application allows to explain and predict the relationship between certain relevant inputs and the results of the achieved effects in the production of pigs. In accordance with the strategy of developing the production of fatteners, the aim of the research is to increase the quality of production in pig breeding with the highest number of analyzed and realized 
economic parameters, originating from noble pure breeds, which are grown on farms in Serbia. When creating this work, data from multiple sources was used. These are the quantities of production, analysis of pig production for a longer period of time. The analysis of such data alone would not be possible if it was not approved by the farm, and the data were further processed by math-statistical methods.

\section{Material and methods}

The research covered the cooperative agricultural farm "1 December" in Žitorađa. The said farm has a closed production cycle that includes the production of fattening pigs. The farm produced 28,829 piglets in 2013. In 2013, 75 persons with appropriate qualifications were employed in the farm. Costs in the production of farm animals on the farm are based on natural indicators determined on the basis of the investigations carried out in 2013. Costs are covered on the basis of the norms of the required space and equipment, approach to the estimation of depreciation costs, on the basis of which the categories of fixed costs are calculated. When determining the surface of a pig, it starts from an independent variable. For conclusions, farm production parameters, costs, increment, total increase and mortality were monitored. The significance of the results of the research in the production of fattening pigs were monitored independently on the farm in 2013.

\section{Results and Discussion}

The strategy of pig production, within the scope of research, was analyzed at the farm: - Planting herds on the farm in 2013,

- Limitations: Accommodation capacities, organizational structure of herds on the farm, - Criteria Function: Minimum Farm Costs.

In addition to theoretical explanation and application on the general examples, the efficiency of the optimal surface on the case of fattening pigs in 2013 on the farm was also shown. The costs of pig production on the farm are based on natural indicators.

In the cooperative household, the calculation of costs was made in accordance with the production processes. Costs also relate to the process of production, space and equipment that is carried out on the basis of norms.

\section{Plantation of a herd on a pig farm "1.Decenbar "in Zitoradza in 2013}

For the pig farming on the farm, the price of all products is taken into account. More information on this is shown in (Tables 1,3,5,7,9 and 11,). The entry status of all categories of pigs for the period from 01.01.to 31.12. 2013.is shown.

Table 2,4,6,8,10 and 12 show the output status of all types of pigs for the period until 31 December 2013. 
Table 1. Input condition

\begin{tabular}{|c|c|c|c|c|c|c|c|c|}
\hline \multirow{3}{*}{ No. } & \multirow{3}{*}{$\begin{array}{l}\text { category } \\
\text { livestock }\end{array}$} & \multicolumn{7}{|c|}{ Admission for the period from 01.01. to 31. 12.2013 } \\
\hline & & \multicolumn{2}{|c|}{$\begin{array}{l}\text { Balance at the } \\
\text { beginningyears }\end{array}$} & \multicolumn{3}{|c|}{ Translated } & \multicolumn{2}{|c|}{ all } \\
\hline & & Who & kg & from the group & Who & kg & Who & kg \\
\hline 1 & 2 & 3 & 4 & 5 & 7 & 8 & 9 & 10 \\
\hline 1. & Prasad & & & Pigs & 7132 & & & \\
\hline 2. & suckling & & & from breeding sows & 657 & & & \\
\hline 3. & Boars & & & from breeding boars & 4 & & & \\
\hline 4. & pigs & 7.132 & & from weaning piglets & 27649 & & 35442 & \\
\hline
\end{tabular}

Source: Authors' calculation based on data from Mičić, 2013

Table 2. Output status

\begin{tabular}{|c|c|c|c|c|c|c|c|c|c|c|}
\hline \multicolumn{11}{|c|}{ Output for the period until 31122013} \\
\hline \multirow{2}{*}{\begin{tabular}{|r|} 
Translated \\
a group \\
\end{tabular}} & \multicolumn{2}{|c|}{ slaughtered } & \multicolumn{2}{|l|}{ died } & \multicolumn{2}{|l|}{ Sold } & \multicolumn{2}{|c|}{$\begin{array}{c}\text { Balance at end } \\
\text { of year }\end{array}$} & \multirow{2}{*}{$\begin{array}{l}\text { all } \\
\mathrm{kg}\end{array}$} & \multirow{2}{*}{$\begin{array}{l}\text { Growth } \\
\text { in kg } \\
23-15\end{array}$} \\
\hline & Who & kg & Who & kg & Who & kg & Who & kg & & \\
\hline 14 & 15 & 16 & 17 & 18 & 19 & 20 & 21 & 22 & 23 & 24 \\
\hline \multicolumn{11}{|l|}{ prefattening } \\
\hline $\begin{array}{l}\text { extracted } \\
\text { sows }\end{array}$ & 186 & 36720 & & & 475 & 107634 & & & & \\
\hline fatling & 6983 & 195080 & 982 & 40980 & 18570 & 1960320 & 7568 & & & \\
\hline $\begin{array}{l}\text { internal } \\
\text { realization }\end{array}$ & 682 & 68200 & & & & & & & & \\
\hline \begin{tabular}{|l|} 
pigs \\
\end{tabular} & & & & & & & 35442 & 2354934 & 3019854 & 664920 \\
\hline
\end{tabular}

Source: Authors' calculation based on data from Mičić, 2013

Table 3. Input condition

\begin{tabular}{|c|c|c|c|c|c|c|c|c|c|}
\hline \multirow{3}{*}{ No. } & \multirow{3}{*}{$\begin{array}{l}\text { category } \\
\text { livestock }\end{array}$} & \multicolumn{8}{|c|}{ Admission for the period from 01.01. to 31. 12.2013 } \\
\hline & & \multicolumn{2}{|c|}{$\begin{array}{l}\text { Balance at the } \\
\text { beginningyears }\end{array}$} & \multirow{2}{*}{$\begin{array}{c}\text { Amount } \\
\text { Rsd }\end{array}$} & \multicolumn{3}{|c|}{ Translated } & \multicolumn{2}{|c|}{ all } \\
\hline & & Who & kg & & $\begin{array}{l}\text { from the } \\
\text { group }\end{array}$ & Who & kg & Who & kg \\
\hline 1 & 2 & 3 & 4 & 5 & 6 & 7 & 8 & 9 & 10 \\
\hline 1. & Prasad suckling & & & & pigs & & & & \\
\hline 2. & pigs1.1.13. & 2858 & 8574 & 1243230 & Prasad & & & & \\
\hline 3. & Oprihodovano & 37642 & & & & & & 40500 & 8574 \\
\hline & In total & 40500 & 8574 & 1243230 & & & & & \\
\hline
\end{tabular}

Source: Authors' calculation based on data from Mičić, 2013 
Table 4. Output status

\begin{tabular}{|c|c|c|c|c|c|c|c|c|c|c|c|c|}
\hline \multicolumn{13}{|c|}{ Output for the period until 31122013} \\
\hline \multicolumn{3}{|c|}{ Translated } & \multicolumn{2}{|c|}{ slaughtered } & \multicolumn{2}{|l|}{ Died } & \multicolumn{2}{|l|}{ Sold } & \multicolumn{2}{|c|}{\begin{tabular}{|c|}
$\begin{array}{c}\text { Balance at end } \\
\text { of year }\end{array}$ \\
\end{tabular}} & \multirow{2}{*}{$\begin{array}{l}\text { all } \\
\text { rsd }\end{array}$} & \multirow{2}{*}{$\begin{array}{l}\text { Growth } \\
\text { in } \mathrm{kg} \\
\mathbf{2 3 - 1 5}\end{array}$} \\
\hline a group & Who & kg & Who & kg & Who & kg & Who & kg & Who & kg & & \\
\hline 12 & 13 & 14 & 15 & 16 & 17 & 18 & 19 & 20 & 21 & 22 & 23 & 24 \\
\hline $\begin{array}{c}\text { In weaned } \\
\text { piglets }\end{array}$ & 31308 & 156540 & & & 6315 & & & & & & 22698300 & \\
\hline $\begin{array}{l}\text { pigs } \\
31.12 .13\end{array}$ & & & & & & & & & 2877 & 8631 & 1251495 & \\
\hline In total & & & & & & & & & 40500 & 165171 & 23949795 & \\
\hline $\begin{array}{l}\text { State } \\
1.1 .13 \\
\end{array}$ & & & & & & & & & 40500 & 8574 & 1243230 & 156597 \\
\hline Growth & & & & & & & & & - & 156597 & 22706565 & \\
\hline
\end{tabular}

Source: Authors' calculation based on data from Mičić, 2013

Table 5. Input condition

\begin{tabular}{|c|c|c|c|c|c|c|c|c|c|}
\hline \multirow{3}{*}{ No. } & \multirow{3}{*}{$\begin{array}{l}\text { category } \\
\text { livestock }\end{array}$} & \multicolumn{8}{|c|}{ Admission for the period from 01.01. to 31. 12.2013 } \\
\hline & & \multicolumn{2}{|c|}{$\begin{array}{l}\text { Balance at the } \\
\text { beginningyears }\end{array}$} & \multirow[b]{2}{*}{ Who } & \multicolumn{3}{|c|}{$\begin{array}{l}\text { Balance at the } \\
\text { beginningyears }\end{array}$} & \multirow[b]{2}{*}{ Who } & \\
\hline & & Who & & & & Who & & & \\
\hline 1 & 2 & 3 & 4 & 5 & 6 & 7 & 8 & 9 & 10 \\
\hline & $\begin{array}{l}\text { weaned } \\
\text { piglets }\end{array}$ & & & & pigs & & & & \\
\hline 1. & pigs 1.1.13. & 4371 & 65565 & 9506925 & Прасад & & & & \\
\hline 2. & $\begin{array}{l}\text { Translated } \\
\text { piglets }\end{array}$ & 31308 & 156540 & 22698300 & & & & 35679 & 222105 \\
\hline 3. & In total & 35679 & 222105 & 32205225 & & & & & \\
\hline
\end{tabular}

Source: Authors' calculation based on data from Mičić, 2013

Table 6. Output status

\begin{tabular}{|c|c|c|c|c|c|c|c|c|c|c|c|c|}
\hline \multicolumn{12}{|c|}{ Output for the period until 31122013} & \multirow{3}{*}{\begin{tabular}{|l} 
Growth \\
in kg \\
$23-15$
\end{tabular}} \\
\hline \multicolumn{3}{|l|}{ Translated } & \multicolumn{2}{|c|}{ slaughtered } & \multicolumn{2}{|l|}{ Died } & \multicolumn{2}{|l|}{ Sold } & \multicolumn{2}{|c|}{$\begin{array}{c}\text { Balance at end } \\
\text { of year }\end{array}$} & \multirow{2}{*}{$\begin{array}{l}\text { all } \\
\text { rsd }\end{array}$} & \\
\hline a group & Who & kg & Who & $\mathrm{kg}$ & Who & $\mathrm{kg}$ & Who & kg & Who & $\mathrm{kg}$ & & \\
\hline 12 & 13 & 14 & 15 & 16 & 17 & 18 & 19 & 20 & 21 & 22 & 23 & 24 \\
\hline Prasad fattening & 27649 & 552980 & & & 3425 & 17125 & & & & & 80182100 & \\
\hline pigs 31.12 .13 & & & & & & & & & 4605 & 73680 & 10683600 & \\
\hline In total & & & & & & & & & 35679 & 643785 & 90865700 & \\
\hline State 1.1.13 & & & & & & & & & 35679 & 222105 & 32205225 & 421680 \\
\hline Growth & & & & & & & & & - & 421680 & 58660475 & \\
\hline
\end{tabular}

Source: Authors' calculation based on data from Mičić, 2013 
Table 7. Input state

\begin{tabular}{|c|c|c|c|c|c|c|c|c|c|}
\hline \multirow{3}{*}{ No. } & \multirow{3}{*}{$\begin{array}{l}\text { Category } \\
\text { Livestock }\end{array}$} & \multicolumn{8}{|c|}{ Entrance for the period from 01.01 .2013} \\
\hline & & \multicolumn{2}{|c|}{$\begin{array}{l}\text { StanjenaBeginning } \\
\text { years }\end{array}$} & \multirow{2}{*}{\begin{tabular}{|r} 
Amount \\
Rsd
\end{tabular}} & \multicolumn{3}{|c|}{ Translated } & \multicolumn{2}{|c|}{ Everything } \\
\hline & & Who & kg & & \begin{tabular}{|l|} 
from the \\
group
\end{tabular} & Who & kg & Who & kg \\
\hline 1 & 2 & 3 & 4 & 5 & 6 & 7 & 8 & 9 & 10 \\
\hline & Breeding gilts & & & & Pigs & & & & \\
\hline 1. & Pigs01.01.2013. & 560 & 73920 & 10718400 & & & & & \\
\hline 2. & $\begin{array}{c}\text { Translated from } \\
\text { the site }\end{array}$ & 682 & 68200 & 9889000 & & & & 1242 & 142120 \\
\hline 3. & In total & 1242 & 142120 & 20607400 & & & & & \\
\hline
\end{tabular}

Source: Authors' calculation based on data from Mičić, 2013

Table 8. Output status

\begin{tabular}{|c|c|c|c|c|c|c|c|c|c|c|c|c|}
\hline \multicolumn{13}{|c|}{ Output for the period until 31122013} \\
\hline \multicolumn{3}{|c|}{ Translated } & \multicolumn{2}{|c|}{ Slaughtered } & \multicolumn{2}{|c|}{ Died } & \multicolumn{2}{|c|}{ Sold } & \multicolumn{2}{|c|}{$\begin{array}{l}\text { Balance at the } \\
\text { end of the year }\end{array}$} & \multirow{2}{*}{\begin{tabular}{|c|}
$\begin{array}{c}\text { Everything } \\
\text { Amount }\end{array}$ \\
rsd \\
\end{tabular}} & \multirow{2}{*}{$\begin{array}{r}\text { Growth } \\
\text { In kg } \\
23-13 \\
\end{array}$} \\
\hline a group & Who & kg & Who & kg & Who & kg & Who & kg & Who & kg & & \\
\hline 12 & 13 & 14 & 15 & 16 & 17 & 18 & 19 & 20 & 21 & 22 & 23 & 24 \\
\hline Sows & 781 & 117150 & & & & & & & & & 16986750 & \\
\hline $\begin{array}{l}\text { Pigs } \\
31.12 .13\end{array}$ & & & & & & & & & 461 & 58335 & 8458575 & \\
\hline In total & & & & & & & & & 1242 & 175485 & 25445325 & \\
\hline $\begin{array}{l}\text { Pigs } \\
01.01 .13\end{array}$ & & & & & & & & & 1242 & 142120 & 20607400 & 33365 \\
\hline Growth & & & & & & & & & - & 33365 & 4837925 & \\
\hline
\end{tabular}

Source: Authors' calculation based on data from Mičić, 2013

Table 9. Input state

\begin{tabular}{|c|c|c|c|c|c|c|c|c|c|}
\hline \multirow[b]{3}{*}{ No. } & \multirow{3}{*}{$\begin{array}{l}\text { Category } \\
\text { Livestock }\end{array}$} & \multicolumn{8}{|c|}{ Entrance for the period from 01.01 .2013} \\
\hline & & \multicolumn{2}{|c|}{\begin{tabular}{|c|} 
Stanjena \\
Beginning years
\end{tabular}} & \multirow{2}{*}{\begin{tabular}{|l|} 
Amount \\
Rsd \\
\end{tabular}} & \multicolumn{3}{|c|}{ Translated } & \multicolumn{2}{|c|}{ Everything } \\
\hline & & Who & kg & & \begin{tabular}{|c|}
$\begin{array}{c}\text { From the } \\
\text { group }\end{array}$ \\
\end{tabular} & Who & kg & Who & kg \\
\hline 1 & 2 & 3 & 4 & 5 & 6 & 7 & 8 & 9 & 10 \\
\hline & Breeding sows & & & & Pigs & & & & \\
\hline 1. & Pigs 01.01.13. & 1534 & 268450 & 38925250 & & & & & \\
\hline 2. & Translated & 781 & 117150 & 16986750 & & & & 2315 & 385600 \\
\hline 3. & In total & 2315 & 385600 & 55912000 & & & & & \\
\hline
\end{tabular}

Source: Authors' calculation based on data from Mičić, 2013 
Table 10. Output status

\begin{tabular}{|c|c|c|c|c|c|c|c|c|c|c|c|c|}
\hline \multicolumn{13}{|c|}{ Output for the period until 31122013} \\
\hline \multicolumn{3}{|c|}{ Translated } & \multicolumn{2}{|c|}{ Slaughtered } & \multicolumn{2}{|c|}{ Died } & \multicolumn{2}{|c|}{ Sold } & \multicolumn{2}{|c|}{$\begin{array}{l}\text { Balance at the } \\
\text { end of the year }\end{array}$} & \multirow{2}{*}{$\begin{array}{c}\text { Everything } \\
\text { Amount }\end{array}$} & \multirow{2}{*}{$\begin{array}{c}\text { Growth } \\
\text { In kg } \\
23-13\end{array}$} \\
\hline a group & Who & kg & Who & kg & Who & kg & Who & kg & Who & kg & & \\
\hline 12 & 13 & 14 & 15 & 16 & 17 & 18 & 19 & 20 & 21 & 22 & 23 & 24 \\
\hline Sows & 657 & 144354 & & & & & & & & & 20931330 & \\
\hline Sows & & & & & 67 & 13930 & & & & & 2019850 & \\
\hline $\begin{array}{l}\text { Pigs } \\
31.12 .13\end{array}$ & & & & & & & & & 1591 & 278425 & 40371625 & \\
\hline In total & & & & & & & & & 2315 & 436709 & 63322805 & \\
\hline $\begin{array}{l}\text { Pigs } \\
01.01 .13\end{array}$ & & & & & & & & & 2315 & 385600 & 55912000 & 51109 \\
\hline Growth & & & & & & & & & - & 51109 & 7410805 & \\
\hline
\end{tabular}

Source: Authors' calculation based on data from Mičić, 2013

Table 11.Input state

\begin{tabular}{|c|l|c|c|c|c|c|c|c|c|}
\hline \multirow{2}{*}{ No. } & \multirow{2}{*}{$\begin{array}{c}\text { Category } \\
\text { Livestock }\end{array}$} & $\begin{array}{c}|c| \\
\text { Stanjena } \\
\text { Beginning } \\
\text { years }\end{array}$ & Amount & \multicolumn{3}{|c|}{ Translated } \\
\cline { 3 - 11 } & & Who & kg & Rsd & $\begin{array}{c}\text { From the } \\
\text { group }\end{array}$ & Who & kg & Who & kg \\
\hline 1 & 2 & 3 & 4 & 5 & 6 & 7 & 8 & 9 & 10 \\
\hline & Breeding nerves & & & & Pigs & & & & \\
\hline 1. & Pigs1.1.13. & 35 & 7770 & 1243200 & & & & & \\
\hline 2. & Purchased nerves & 6 & 771 & 253704 & & & & 41 & 8541 \\
\hline 3. & In total & 41 & 8541 & 1380354 & & & & & \\
\hline
\end{tabular}

Source: Authors' calculation based on data from Mičić, 2013 
Table 12. Output status

\begin{tabular}{|c|c|c|c|c|c|c|c|c|c|c|c|c|c|}
\hline \multicolumn{10}{|c|}{ Output for the period until 31 12 2013 } \\
\hline \multicolumn{2}{|c|}{ Translated } & $\begin{array}{c}\text { Slaughte } \\
\text { red }\end{array}$ & \multicolumn{2}{|c|}{ Died } & \multicolumn{2}{c|}{ Sold } & $\begin{array}{c}\text { Balance at } \\
\text { the end of the } \\
\text { year }\end{array}$ & $\begin{array}{c}\text { Everything } \\
\text { Amount }\end{array}$ & $\begin{array}{c}\text { Growth } \\
\text { In kg } \\
\mathbf{2 3 - 1 3}\end{array}$ \\
\hline a group & Who & kg & Who & kg & Who & kg & Who & kg & Who & kg & rsd & \\
\hline 12 & 13 & 14 & 15 & 16 & 17 & 18 & 19 & 20 & 21 & 22 & 23 & 24 \\
\hline Unruly & 4 & 1320 & & & & & & & & & 191400 & \\
\hline Unruly & & & & & 3 & 890 & & & & & 129050 & \\
\hline Pigs 31.12.2013 & & & & & & & & & 34 & 8500 & 1232500 & \\
\hline In total & & & & & & & & & 41 & 10710 & 1552950 & \\
\hline Pigs01.01.2013 & & & & & & & & & 41 & 8541 & 1380354 & 2169 \\
\hline Growth & & & & & & & & & - & 2169 & 172596 & \\
\hline
\end{tabular}

Source: Authors' calculation based on data from Mičić, 2013

Note: Table 1,3,5,7,9 and 11 shows the entry status of all types of pigs for the period from01.01 to 31.12 .2013 . Table $2,4,6,8,10$ and 12 show the output status of all types of pigs for the period until 31 December 2013.

Table 13.Livestock income on farm for 2013.

\begin{tabular}{|l|c|c|}
\hline \multicolumn{1}{|c|}{ Category of livestock } & Kg & Amount (€) \\
\hline Piglets on cheese & 156.597 & $189.221,4$ \\
\hline Closed piglets & 421.680 & $488.837,3$ \\
\hline Breeding gilts & 33.365 & $40.316,0$ \\
\hline Breeding sows & 51.109 & $61.756,7$ \\
\hline Breeding nerves & 2.169 & $1.438,3$ \\
\hline Total increase & 664.920 & $781.569,7$ \\
\hline
\end{tabular}

Source: Authors’ calculation based on data from Mičić, 2013

Table 14.Will end up at the farm for 2013.

\begin{tabular}{|c|c|c|c|c|c|c|c|c|c|}
\hline $\begin{array}{l}\text { January- } \\
\text { December }\end{array}$ & $\begin{array}{l}\text { Piglets on } \\
\text { cheese }\end{array}$ & \multicolumn{2}{|c|}{ Closed piglets } & \multicolumn{2}{|c|}{ Running pigs } & \multicolumn{2}{|c|}{ Breeding sows } & \multicolumn{2}{|c|}{$\begin{array}{c}\text { Breeding } \\
\text { nerves }\end{array}$} \\
\hline Moon & Who & Who & kg & Who & kg & Who & kg & Who & kg \\
\hline I & 515 & 270 & 5 & 141 & 6140 & 7 & 1490 & & \\
\hline II & 510 & 280 & 5 & 106 & 4750 & 4 & 850 & & \\
\hline III & 550 & 290 & 5 & 118 & 4570 & 4 & 860 & & \\
\hline IV & 540 & 280 & 5 & 79 & 3300 & 5 & 1070 & 3 & 890 \\
\hline $\begin{array}{l}\text { January- } \\
\text { December }\end{array}$ & $\begin{array}{l}\text { Piglets on } \\
\text { cheese }\end{array}$ & \multicolumn{2}{|c|}{ Closed piglets } & \multicolumn{2}{|c|}{ Running pigs } & \multicolumn{2}{|c|}{ Breeding sows } & \multicolumn{2}{|c|}{$\begin{array}{c}\text { Breeding } \\
\text { nerves }\end{array}$} \\
\hline Moon & Who & Who & kg & Who & kg & Who & kg & Who & kg \\
\hline
\end{tabular}




\begin{tabular}{|c|c|c|c|c|c|c|c|c|c|}
\hline V & 520 & 320 & 5 & 84 & 3420 & 3 & 630 & & \\
\hline VI & 540 & 285 & 5 & 67 & 2760 & 6 & 1230 & & \\
\hline VII & 510 & 290 & 5 & 64 & 2670 & 10 & 2040 & & \\
\hline VIII & 510 & 285 & 5 & 50 & 2140 & 7 & 1420 & & \\
\hline IX & 540 & 300 & 5 & 63 & 2590 & 3 & 630 & & \\
\hline X & 540 & 285 & 5 & 64 & 2690 & 6 & 1200 & & \\
\hline XI & 530 & 270 & 5 & 69 & 2960 & 5 & 1020 & & \\
\hline XII & 510 & 270 & 5 & 77 & 2990 & 7 & 1490 & & \\
\hline In total & 6.315 & 3.425 & 17.150 & 982 & 40.980 & 67 & 13.930 & 3 & 890 \\
\hline
\end{tabular}

Source: Authors' calculation based on data from Mičić, 2013

Note: Table 13 shows the increase in livestock of all types of pigs for the period from $01 / 01 / 2013$ to $31 / 12 / 2013$, and in Table 14, the cattle will die in the farm in 2013

\section{Setting a model to solve a defined problem}

When setting the model, we first define the production lines that take into account to enter the optimal production structure.

In the model model, it starts from the assumption that in the selected livestock capacities the optimal organizational structure of the flock of a pig farmer's net effective area of $12000 \mathrm{~m}^{2}$, by linear programming, is determined.

The organizational structure should be framed within the following limits:

Piglets 20-25\%

Breeding subspecies 5-8\%

Fattening pigs $70-80 \%$

Sows $4-8 \%$ i

Unbreakable 0,3-5\%.

The following space is planned for the individual of certain categories: piglets on cheese $0,4 \mathrm{~m}^{2}$, breeding pig $0,7 \mathrm{~m}^{2}$, pig fodder $1,2 \mathrm{~m}^{2}$, sowers $1,5 \mathrm{~m}^{2}$ and nests $1,8 \mathrm{~m}^{2}$.

The costs of individual holding categories are as follows:

For a piglet 700 dinars, piglets for breeding (breeding subspecies) 1,800 dinars, pig meat 3,500 dinars, sowers 5,000 dinars and non-dinner 4,000 dinars.

In our case, it is defined through the number of livestock category cattle, accommodation capacities, organizational structure of herds and direct costs per unit of capacity.

In solving the model, it is necessary to define the economic function of the criteria. 
1. Independent variable:

$\mathrm{Xi}$ - number of livestock category cattle "and"

$\mathrm{I}=1$ (1) 5 - cattle categories

$\mathrm{I}=1$ - piglets

$\mathrm{I}=2$ - breeding subspecies

$\mathrm{I}=3$ - fattening pigs

$\mathrm{I}=4$ - sows

$\mathrm{I}=5$ - unbreakable

2. Limitations:

a) Accommodation capacities

$0,4 \mathrm{~m} 2$ / throat X1 (throat) $+0,7 \mathrm{X} 2+1,2 \mathrm{X} 3+1,5 \mathrm{X} 4+1,8 \mathrm{X} 5=12,000 \mathrm{~m}^{2}$

b) Organizational structure of the herd

$\mathrm{X} 1>0,20(\mathrm{X} 1+\mathrm{X} 2+\mathrm{X} 3+\mathrm{X} 4+\mathrm{X} 5) ; 0,80 \mathrm{X} 1-0,20(\mathrm{X} 2+\mathrm{X} 3+\mathrm{X} 4+\mathrm{X} 5)>0$

$\mathrm{X} 1<0,25(\mathrm{X} 1+\mathrm{X} 2+\mathrm{X} 3+\mathrm{X} 4+\mathrm{X} 5) ; 0,75 \mathrm{X} 1-0,25(\mathrm{X} 2+\mathrm{X} 3+\mathrm{X} 4+\mathrm{X} 5)<0$

$\mathrm{X} 2>0,05(\mathrm{X} 1+\mathrm{X} 2+\mathrm{X} 3+\mathrm{X} 4+\mathrm{X} 5) ; 0,95 \mathrm{X} 2-0,05(\mathrm{X} 1+\mathrm{X} 3+\mathrm{X} 4+\mathrm{X} 5)>0$

$\mathrm{X} 2<0,08(\mathrm{X} 1+\mathrm{X} 2+\mathrm{X} 3+\mathrm{X} 4+\mathrm{X} 5) ; 0,92 \mathrm{X} 2-0,08(\mathrm{X} 1+\mathrm{X} 3+\mathrm{X} 4+\mathrm{X} 5)<0$

$\mathrm{X} 3>0,7(\mathrm{X} 1+\mathrm{X} 2+\mathrm{X} 3+\mathrm{X} 4+\mathrm{X} 5) ; 0,3 \mathrm{X} 3-0,7(\mathrm{X} 1+\mathrm{X} 2+\mathrm{X} 4+\mathrm{X} 5)>0$

$\mathrm{X} 3<0,8(\mathrm{X} 1+\mathrm{X} 2+\mathrm{X} 3+\mathrm{X} 4+\mathrm{X} 5) ; 0,2 \mathrm{X} 3-0,8(\mathrm{X} 1+\mathrm{X} 2+\mathrm{X} 4+\mathrm{X} 5) 0$

$\mathrm{X} 4>0,04(\mathrm{X} 1+\mathrm{X} 2+\mathrm{X} 3+\mathrm{X} 4+\mathrm{X} 5) ; 0,96 \mathrm{X} 4-0,04(\mathrm{X} 1+\mathrm{X} 2+\mathrm{X} 3+\mathrm{X} 5)>0$

$\mathrm{X} 4<0,08(\mathrm{X} 1+\mathrm{X} 2+\mathrm{X} 3+\mathrm{X} 4+\mathrm{X} 5) ; 0,92 \mathrm{X} 4-0,08(\mathrm{X} 1+\mathrm{X} 2+\mathrm{X} 3+\mathrm{X} 5)<0$

$\mathrm{X} 5>0,003(\mathrm{X} 1+\mathrm{X} 2+\mathrm{X} 3+\mathrm{X} 4+\mathrm{X} 5) ; 0,997 \mathrm{X} 5-0,003(\mathrm{X} 1+\mathrm{X} 2+\mathrm{X} 3+\mathrm{X} 4)>0$

$\mathrm{X} 5<0,05(\mathrm{X} 1+\mathrm{X} 2+\mathrm{X} 3+\mathrm{X} 4+\mathrm{X} 5) ; 0,95 \mathrm{X} 5-0,05(\mathrm{X} 1+\mathrm{X} 2+\mathrm{X} 3+\mathrm{X} 4)<0$

3. Function of criteria:

Minimum expenses

$700 \mathrm{din} /$ throatX1 (throat) $+1,800 \times 2+3,500 \times 3+5,000 \times 4+4,000 \times 5$ $=\mathrm{V}(\min )$

In setting up the model of the optimal structure of the household, one of the most important issues is defining the factors over the corresponding ones Which will, through the linear programming model, essentially influence the flow of the solution and the selection of the structure of pig production.

Projection of the optimization of the structure of pig production is based on the achieved 
average production and economic results in order to compare the projected and actual production structure and economic results.

Task solution:

MIN

$700 \times 1+1800 \times 2+3500 \times 3+5000 X 4+4000 \times 5$

SUBJECT TO

$0.4 \mathrm{X} 1+0.7 \mathrm{X} 2+1.2 \mathrm{X} 3+1.5 \mathrm{X} 4+1.8 \mathrm{X} 5=12000$

$0.80 \mathrm{X} 1-0.20 \mathrm{X} 2-0.20 \mathrm{X} 3-0.20 \mathrm{X} 4-0.20 \mathrm{X} 5>0$

$0.75 \mathrm{X} 1-0.25 \mathrm{X} 2-0.25 \mathrm{X} 3-0.25 \mathrm{X} 4-0.25 \mathrm{X} 5<0$

$0.95 \mathrm{X} 2-0.05 \mathrm{X} 1-0.05 \mathrm{X} 3-0.05 \mathrm{X} 4-0.05 \mathrm{X} 5>0$

$0.92 \mathrm{X} 2-0.08 \mathrm{X} 1-0.08 \mathrm{X} 3-0.08 \mathrm{X} 4-0.08 \mathrm{X} 5<0$

$0.3 \mathrm{X} 3-0.7 \mathrm{X} 1-0.7 \mathrm{X} 2-0.7 \mathrm{X} 4-0.7 \mathrm{X} 5>0$

$0.2 \mathrm{X} 3-0.8 \mathrm{X} 1-0.8 \mathrm{X} 2-0.8 \mathrm{X} 4-0.8 \mathrm{X} 5<0$

$0.96 \mathrm{X} 4-0.04 \mathrm{X} 1-0.04 \mathrm{X} 2-0.04 \mathrm{X} 3-0.04 \mathrm{X} 5>0$

$0.92 \mathrm{X} 4-0.08 \mathrm{X} 1-0.08 \mathrm{X} 2-0.08 \mathrm{X} 3-0.08 \mathrm{X} 5<0$

$0.997 \mathrm{X} 5-0.003 \mathrm{X} 1-0.003 \mathrm{X} 2-0.003 \mathrm{X} 3-0.003 \mathrm{X} 4>0$

$0.95 \times 5-0.05 X 1-0.05 X 2-0.05 X 3-0.05 X 4<0$

END

LP OPTIMUM FOUND AT STEP 6

OBJECTIVE FUNCTION VALUE

$0.2575503 \mathrm{E}+08$

VARIABLE VALUE REDUCED COST

$\begin{array}{rrr}\text { X1 } & 2516.778564 & 0.000000 \\ \text { X2 } & 335.570465 & 0.000000 \\ \text { X3 } & 5033.557129 & 0.000000 \\ \text { X4 } & 419.463074 & 0.000000 \\ \text { X5 } & 83.892616 & 0.000000\end{array}$


ROW SLACK OR SURPLUS DUAL PRICES

$\begin{array}{lrr}2) & 0.000000 & -2575.503418 \\ 3) & 419.463074 & 0.000000 \\ 4) & 0.000000 & 427.348999 \\ 5) & 83.892616 & 0.000000 \\ 6) & 83.892616 & 0.000000 \\ 7) & 0.000000 & -197.147644 \\ 8) & 838.926147 & 0.000000 \\ 9) & 0.000000 & -1424.496704 \\ 10) & 2516.778564 & 0.000000 \\ 11) & 41.946312 & 0.000000 \\ 12) & 0.000000 & 90.604027\end{array}$

RANGES IN WHICH THE BASIS IS UNCHANGED:

OBJ COEFFICIENT RANGES

VARIABLE CURRENT ALLOWABLE ALLOWABLE

COEF INCREASE DECREASE

$\begin{array}{lrrr}\text { X1 } & 800.000000 & 397.347900 & 300.000000 \\ \text { X2 } & 2000.000000 & 192.622940 & 87.096779 \\ \text { X3 } & 4000.000000 & 2830.000000 & 150.000000 \\ \text { X4 } & 6000.000000 & 6714.285645 & 1486.865234 \\ \text { X5 } & \text { 5000.000000 } & 91.525421 & \text { INFINITY } \\ & \text { RIGHTHAND } & \text { SIDE RANGES } & \end{array}$

ROW CURRENT ALLOWABLE ALLOWABLE

RHS INCREASE DECREASE

$\begin{array}{lrll}2 & 10000.000000 & \text { INFINITY } & 9999.999023 \\ 3 & 0.000000 & 419.463074 & \text { INFINITY } \\ 4 & 0.000000 & 84.104294 & 83.682007 \\ 5 & 0.000000 & 83.892616 & \text { INFINITY } \\ 6 & 0.000000 & \text { INFINITY } & 83.892616\end{array}$




\begin{tabular}{llrr}
\hline 7 & 0.000000 & 83.402832 & \multicolumn{1}{c}{84.388184} \\
8 & 0.000000 & INFINITY & 838.926147 \\
9 & 0.000000 & 83.194679 & 84.602364 \\
10 & 0.000000 & INFINITY & 2516.778564 \\
11 & 0.000000 & 41.946312 & INFINITY \\
12 & 0.000000 & 83.056480 & 42.158520
\end{tabular}

\section{Conclusion}

Based on the analysis of the situation in the production and the optimal organizational structure of the livestock farm, which ensures minimal costs in the amount of 25,755,000 dinars, the following structure of the herd has been achieved: 2.517 heads of pigs, 336 seeds of juvenile breeding, pigs 5.034 heads, 419 sows and nerazoles 84 throats. Total deaths on the farm in 2013 were: pigs on cheese 6,315 heads, 3,425 heads, 17,150 kg pigs, 988 heads, 40,980 kg pigs, 67 seeds, 13,930 kg breeders and $890 \mathrm{~kg}$ of unadjusted 3 heads. The total increase in livestock per farm for the first year is $664.920 \mathrm{~kg}$, from $781.569,7$ EUR. Another argument in the request for determining the costs of pig production according to the meat share, which was shown in the farm's research, is that it is best to close the entire production cycle. This is primarily the market for fattening pigs, piglets and pig meat in Serbia in 2013. After the research, a number of very important questions were answered in the paper: the level of costs is not such as to stimulate pig farmers, and the price affects the volume of production. Consumption of pork is cyclically reduced due to relatively high prices and a decline in living standards in the Republic of Serbia. The new scientific findings in pig breeding have come to light, the advantages of this method of training have been examined and scientific contribution has been given to the improvement of the development of pig production, for which Serbia, along with pig breeding tradition, has excellent geographical ecological potentials. As the final conclusion of these studies, pig farms may be recommended to be organized into production groups, co-operatives, clusters or franchisees (franchisees) or franchisees (franchises) to be able to place their products at higher prices. The basis for achieving better production results is a change in the type of production, meals, facilities, mechanization of work processes, farm size, intensity, rational management of costs in farm conditions. Because of their specificity, primarily biological character, each line of agricultural production must be viewed and organized as a complex process composed of a number of elements having one goal, namely achieving as good a result as possible with a lower investment unit. In Serbian swineyard, there is a traditionalism that needs to undergo fundamental changes in order to achieve the desired results. Such results can be achieved only in the case of the establishment of a fully functional closed system of breeding. There is a traditionalism in Serbian swine that needs to go through fundamental changes in order to achieve the desired results. Such results can be achieved only in the case of the establishment of a fully functional closed herd breeding system. 


\section{Literature}

1. Andrić, J. (1998): Troškovii kalkulacije u poljoprivrednoj proizvodnji, Poljoprivredni fakultet - Zemun, Beograd; CouncilRegulation (EEC) No 3220/84 of 13 November 1984 determiningthe Community scale forgrading pig carcass, 1984. Official Journal ofthe European Communities No L 301, 20/11/1984, No. $1-3$.

2. Džinić, N., Petrović, Lj., Tomović, V., Manojlović, D., Timanović, S., Trišić-Ilić, S., Mrdjanov, J. (2003): Uticaj višerasnog ukrštanja i godišnjeg doba na kvalitet polutki i mesa svinja, Savremena poljoprivreda, Vol. 52, No. 3-4, pp. 335-337.

3. Džinić, N., Petrović, Lj.,Tomović, V., Manojlović, D., Timanović, S., Vidanrić, D., Kurjakov, N. (2004): Ocena kvaliteta polutki i mesa svinja rasa veliki švedski ladras. Biotechnology in animal husbandry, Vol. 20, No. 1 1-2, pp. 67-73.

4. Fisher, A.V., Green, D.M., Whittemore, C.T., Wood, J.D., Schofield, C.P. (2003): Growth of carcass components and its relation with conformation in pigs of threetypes, Meat Science, Vol. 65, No. 1, pp. 639-650.

5. Jukna, V., Jukna, C. (2005): The comparable estimation of meat quality of pigsbreeds and their combinations in Lithuania, Biotechnology in Animal Husbandry, Vol. 21, No. 5-6, pp. 175-179.

6. Kosovac, O., Josipović, S., Živković, B., Radović, Č., Marinkov, G., Tomašević,D. (2007): Comparable presentation of carcass and meat quality of differentpig genotypes using modern evaluation methods, . 2nd International Congresson animal husbandry „New perspectives and challenges of sustainable livestockfarming”, Belgrade, Serbia, Biotechnology In Animal Husbandry, Vol. 23, No. 5-6, pp. 291301.

7. Manojlović, D., Petrović, Lj., Džinić, N., Kurjakov, N. (1999): Kvalitet trupa $i$ mesa svinja - osnova kvaliteta proizvoda, Monografija: Tehnologija proizvodnje i kvalitet konzervi od mesa u komadima, No. 67-90, Tehnološki fakultet u Novom Sadu.

8. Mičić, I. (2014): Strategija razvoja proizvodnje svinjskog mesa i njegova ekonomska opravdanost, magistarska teza, Poljoprivredni fakultet, Univerzitet u Prištini, Srbija.

9. Mičić, I., Urošević, D., Vujić, R., Mičić, I. I., Mičić, M., Mičić, I. M. (2016a): Operating costs of agricultural holdings with equal production possibilities, Economics of Agriculture Belgrade Serbia, Vol. 63, No. 2, pp. 407-428.

10. Mičić, I., Rajić, Z., Živković, D., Nikolić, S., Mičić, I. I., Mičić, I. M. (2016b): Development strategy of economically justified pig meat production from farm to fork: agricultural economic analysis, ZAEM, Association of Agricultural Economists of Republic of Macedonia, AAEM $10^{\mathrm{TH}}$ Intrnational Conference, UDC 338.439.4:637.5'64:303.72 (497.11) pp. 148-155.

11. Petrović, M., Radović, Č., Perunović, N., Radojković, D., Aleksić, S., Stanišić, N., Popovac, N. (2010): Quality traits of carcass sides and meat of moravka and mangalitsa pig breeds, Biotechnology in Animal Husbandry, Vol. 26, pp. 21-27. 
12. Petrović, M. M., Aleksić, S., Petrović, M. P., Petrovič, M., Pantelić, V., Novaković, Ž., Ružić Muslić, D. (2013): Potencijali stočarske proizvodnje, Belgrad, Srbia, Biotechnology in Animal Husbandry Vol. 29, No. 1, pp 1-17.

13. Radović, B. (2000): Gajenje svinja, D. P. 'Grafika”, Kruševac.

14. Radović, I. (2002): Primena linearnog programiranja u poljoprivredi, Potez, Beograd.

15. Rahelić, S. (1984): Uzgoj svinje i meso, Školska knjiga, Zagreb.

16. Rajić, Z. (2003): Model za optimiranje structure proizvodnje industrijske proizvodnje klanice, doktorska disertacija, Poljoprivredni fakultet, Univerzitet u Novom Sadu, Srbija.

17. Timanović, S., Vidanić, D., Kurjakov, N. (2003): Ocena kvaliteta polutki i mesa svinja rasa veliki jorkšir i švedski landras, Biotehnologija u stočarstvu Vol. 19, No. 5-6, p. 500.

18. Tomović, V., Petrović, Lj., Džinić, N.,Manojlović, D.,Timanović, S., TrišićIlić, S. (2005): Meat yield in primal cuts and sides of pigs determined by partial dissection, International 53rd meat industry conference "New integral approach to meat andproducts safety", Vrnjačka banja, I-15, No. 31-34.

19. Vidović, V., Krnjaić, J., Lukač, D., Višnjić, V., Stupar, M. (2012): Growth intensity of the gilts fertile breed pigs in the Nucleus farm, Biotech, Anim. Hus., Vol. 28, No. 4, pp. 787-796.

20. Zekić, V., Okanović, Đ. (2007): Prudence of fatty pigs production, I International Congress: „FOOD TECHNOLOGY, QUALITY AND SAFETY“, XI Symposium NODA: „Technology, quality andsafety in pork production and meat Processing“, Proceedings No. 33-37.

21. Zekić, V., Tomović, V., Milić, D., Vidović, V., Lukač, D. (2013): Ekonomska obeležja proizvodnje svinja rase landras $i$ mangulica, Letopis naučnih radova Poljoprivrednog fakulteta, Vol. 37, No. 1, pp. 191-199.

22. Živković, D., Perunović, M. (2012): Poznavanje mesa, praktikum, Poljoprivredni fakultet, Univerzitet u Beogradu. 


\title{
OPTIMALNA STRUKTURA SVINJA NA FARMI SA MINIMALNIM PROIZVODNIM TROŠKOVIMA
}

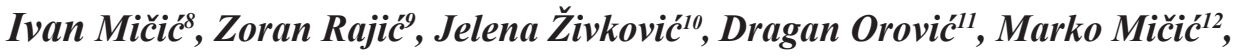 \\ Ivana Mičićc ${ }^{13}$, Marija Mičić ${ }^{14}$
}

\section{Rezime}

Studija se bavi analizom obrta stada svinjogojske farme u proizvodnji svinjskog mesa u Srbiji, kao i neto efektivne površine oprimalne organizacione structure tovljenika u farmskim uslovima. Postavlja se pitanje optimalne structure proizvodnje na zadružnom gazdinstvu, koja je uglavnom orijentisano na proizvodnji tovnih svinja. $U$

radu je definisan i urađen konkretan model linearnog programiranja za optimalnu organizacionu strukturu stada svinjarske farme neto efektivne površin., Istraživanje je obuhvatilo zadružnu farmu „1. Decembr" u Žitorađi u Topličkom okrugu. Za 2013 godinu praćena je tehnologija proizvodnje tovljenika na farmi, i analizirani su ekonomski rezultati. Tokom jednogodišnjeg posmatranog perioda.Utvrđeno je da je na farmi uzgoj tovljenika bio je 28.252 grla. Ukupni prirast na farmi, u 2013. godini bio je $664.920 \mathrm{~kg}$, u iznosu od 781.569,7 €. Ukupno uginuće: prasadi na sisi 6.315 grla, zalučena prasad 3.425 grla, $17.150 \mathrm{~kg}$, tovnih svinja $928 \mathrm{grla}, 40.980 \mathrm{~kg}$, krmače 67 grla $13.930 \mathrm{~kg}$, nerastova 3 grla $890 \mathrm{~kg}$.

Ključne reči: obrt stada, površine po grlu, prirast, uginuće, minimalni troškovi

8 Mr Ivan Mičić, doktorant, Univerzitet u Beogradu, Poljoprivredni fakultet, Nemanjina ulica br. 6, 11080 Zemun, Srbija, Telefon: +381 6297311 58, E-mail: divanlav@gmail.com

9 Redovni profesor, dr Zoran Rajić, Univerzitet u Beogradu, Poljoprivredni fakultet, Nemanjina ulica br. 6, 11080 Zemun, Srbija, Telefon: +381 1126153 15, E-mail: zorajic@agrif.bg.ac.rs

10 Jelena Živković, Master, Univerzitet u Beogradu, Poljoprivredni fakultet, Nemanjina ulica br. 6, 11080 Zemun, Srbija, Telefon: +381 1126153 15, E-mail: zivkovic@yahoo.com

11 Mr Dragan Orović, doktorant, Univerzitet u Beogradu, Poljoprivredni fakultet, Nemanjina ulica br. 6, 11080 Zemun, Srbija, Telefon: +381 6987025 20, E-mail: draganorovic@gmail.com

12 Marko Mičić, diplomirani ekonomista, Univerzitet u Nišu, Ekonomski fakultet, Trg Kralja Aleksandra Ujedinitelja br. 11, 18000 Niš, Srbija, Telefon: +381 63680 040, E-mail: markomicic89@gmail.com

13 Ivana Mičić M.A., doktorand, Univerzitet u Nišu, Ekonomski fakultet, Trg Kralja Aleksandra Ujedinitelja br. 11, 18000 Niš, Srbija, Telefon: +381 63233 603, E-mail: ivancica@gmail.com

14 Marija Mičić M.A., doktorant, Univerzitet u Nišu, Tehnološki fakultet u Leskovcu, Bulevar Oslobođenja br. 124, 16000 Leskovac, Srbija, Telefon: +381 62867 45 98, E-mail: marija84micic@gmail.com 